\title{
Definição de Processos Reutilizáveis para Projetos com Aquisição
}

\author{
Fabrício Souto Cardoso ${ }^{1,2}$, Ana Regina Rocha ${ }^{1}$ \\ ${ }^{1}$ COPPE/UFRJ - Universidade Federal do Rio de Janeiro \\ Caixa Postal 68511 - CEP 21945-970, Rio de Janeiro, Brasil \\ ${ }^{2}$ ELETROBRAS - Centrais Elétricas Brasileiras S.A.
}

Av. Presidente Vargas, 409/16 ${ }^{\circ}$ andar, Centro - CEP 20071-003, Rio de Janeiro, Brasil

\{fasouto, darocha\}@cos.ufrj.br, fabricio.cardoso@eletrobras.com

\begin{abstract}
It is possible to find in the literature researches which demonstrate the high degree of failure, risks and problems arising from the acquisition model in software development projects. This paper aims to present, in general, the approach used for the definition of reusable process components, built using features present in organizations with software acquisition development projects, which were made available in a Library of Reusable Processes Components. Thus, it is expected to facilitate the processes definition in public or private organizations that acquire software, reducing the cost and effort associated with this activity.
\end{abstract}

Resumo. Podemos encontrar na literatura pesquisas que demonstram o alto grau de insucesso, riscos e problemas advindos do modelo de aquisição em projetos de desenvolvimento de software. Este trabalho tem como objetivo apresentar, em linhas gerais, a abordagem utilizada durante a definição de componentes de processo reutilizáveis, construídos utilizando características presentes em organizações que possuem projetos de desenvolvimento de software com aquisição, os quais foram disponibilizados em uma Biblioteca de Componentes Reutilizáveis de Processos. Dessa forma, espera-se facilitar a definição de processos em organizações públicas ou privadas que adquirem software, diminuindo o custo e o esforço associado a essa atividade.

\section{Introdução}

Em busca por vantagem competitiva, muitas organizações estão optando, cada vez mais, pelo modelo de aquisição ou terceirização de seus serviços de Tecnologia da Informação (TI), com o objetivo de melhorar sua eficiência operacional, através da especialização e capacitação de seus fornecedores no provimento desses serviços [SEI, 2010]. Contudo, não é difícil encontrar na literatura autores e pesquisas que demonstrem o alto grau de insucesso, riscos e problemas advindos dessas iniciativas [Khan et al., 2009; Nunes, 2011]. Essas mesmas pesquisas indicam que a não utilização de processos adequados representa uma das causas para o insucesso de projetos de desenvolvimento de software com aquisição.

No que se refere à pertinência e relevância de processos de software adequados às contratações feitas por órgãos públicos brasileiros, o TCU (Tribunal de Contas da União) estabelece que qualquer organização pública que pretenda contratar serviços de 
software precisa ter processos internos com qualidade compatível com aquela demandada do contratado, a fim de garantir a efetividade do contrato [TCU, 2010].

Contudo, definir um processo de software não é uma atividade simples, pois exige, além da experiência dos profissionais envolvidos, a harmonização de diversos aspectos e conhecimentos existentes na engenharia de software. Ainda no contexto da definição de processos, pesquisas demonstram que uma das principais práticas para prover sua melhoria contínua é a utilização de técnicas de reutilização, pois possibilitam o aproveitamento de informações e medições coletadas em projetos já finalizados ou em andamento, reduzindo o esforço necessário a ser aplicado em novos projetos.

Portanto, este artigo tem como objetivo apresentar um resumo do trabalho de dissertação de mestrado realizado por CARDOSO [2012], onde espera-se que organizações públicas ou privadas que almejam definir processos em conformidade com o MR-MPS-SW e que possuam projetos de desenvolvimento de software com aquisição (terceirização das atividades relacionadas ao desenvolvimento de software), possam diminuir o custo e o esforço associado a essa atividade, tornando, inclusive, sua realização acessível a profissionais menos experientes, mediante a reutilização dos elementos de processo nele disponibilizados e apresentados neste artigo.

Dessa forma, a seção 2 apresenta os principais conceitos relacionados à técnica que foi utilizada na definição de processos para reutilização, bem como a justificativa para a escolha da mesma. Na seção 3 é apresentada a abordagem seguida para a definição de elementos de processos reutilizáveis voltados a organizações que possuam projetos de desenvolvimento de software com aquisição, utilizando as características presentes em tais organizações. Tais elementos foram disponibilizados no trabalho de CARDOSO [2012] e em uma Biblioteca de Componentes Reutilizáveis de Processos já existente [Barreto, 2011] e mantida pelo grupo de Qualidade de Software da COPPE/UFRJ. As considerações finais e as contribuições do trabalho são apresentadas na seção 4. Até este momento, a biblioteca está populada, apenas, com elementos de processo que atendem aos níveis $\mathrm{G}$ e $\mathrm{F}$ do MR-MPS-SW, o que atende atualmente $100 \%$ das organizações alvo deste trabalho. Pretende-se, no futuro, que sejam definidos componentes que atendam aos requisitos dos demais níveis de maturidade.

\section{Técnica Utilizada para Reutilização de Processos de Software}

\subsection{Justificativa para Escolha da Técnica}

Para a realização do trabalho de definição de processos para reutilização foi utilizada a técnica definida por BARRETO [2011]. Essa técnica adapta conceitos normalmente utilizados na reutilização de produtos de software para o contexto da definição de processos, modelando os conceitos envolvidos, estabelecendo como definir processos reutilizáveis e como, a partir destes, derivar outros processos. Além disso, considera requisitos relacionados à definição de processos em organizações de alta maturidade e disponibiliza apoio ferramental para realização de suas principais etapas.

Com relação ao apoio ferramental oferecido, a abordagem escolhida conta com o apoio de uma ferramenta para definição de componentes e linhas de processos, desenvolvida e integrada ao ambiente A2M (Ambiente de Alta Maturidade), desenvolvido pela COPPE/UFRJ, como forma de apoiar a realização de práticas de alta maturidade. Outro trabalho que colaborou para a escolha dessa técnica foi o realizado 
por NUNES [2011], que definiu componentes de processos e uma linha de processos de software para o processo Aquisição (AQU), disponibilizando-os na biblioteca de componentes contida na referida ferramenta, possibilitando que algumas características e componentes já existentes pudessem ser reutilizados.

Segundo resultados obtidos no estudo experimental realizado no âmbito do trabalho de BARRETO [2011], a utilização de linhas de processo pode reduzir significativamente o esforço e o tempo necessários para definir processos para um projeto. Essa pesquisa identificou, também, que o tempo para derivação de um processo definido para um projeto utilizando linhas de processo foi, aproximadamente, $25 \%$ mais rápido do que utilizando somente componentes. Em pesquisas de opinião aplicadas antes e após o referido estudo, os resultados apontaram que a expectativa por benefícios obtidos ao utilizar tal abordagem aumentou, se comparada a outras técnicas de reutilização de processos.

\subsection{Principais Conceitos Relacionados}

Um dos principais conceitos presentes na técnica definida por BARRETO [2011] é o de "Elemento de Processo". Ele é definido como sendo um encapsulamento de informações e comportamentos de processo em um dado nível de granularidade, representando uma ação de transformação realizada por um processo. Esse conceito pode ser especializado em dois outros: "Componente de Processo" e "Atividade". Contudo, o nível de granularidade a ser utilizado na definição desses elementos irá depender do uso pretendido.

Apesar dessa flexibilidade, BARRETO [2011] estabelece algumas características importantes que devem estar presentes em um elemento de processo para que o mesmo possa ser considerado um componente, tais como: (i) ser relevante para ser reutilizado; (ii) representar um subprocesso relevante de um processo de mais alto nível, podendo ser realizado de uma ou várias maneiras; (iii) ser relevante para ser medido e ter sua estabilidade e desempenho analisados; (iv) atuar como um container que encapsula outros elementos de processo, através de sua decomposição em uma arquitetura de processos interna; (v) admitir variabilidades, ou seja, poder representar diferentes maneiras de realizar uma parte de um processo.

Esses "Componentes de Processo" podem ser especializados em "Componentes de Processo Abstratos" e "Componentes de Processo Concretos". Um "Componente de Processo Concreto" é aquele que não admite qualquer variação, sendo executado da forma com que foi descrito. Ele pode ser utilizado diretamente em um projeto, pois não há mais nenhum elemento de processo, presente em sua arquitetura interna, que dependa de alguma decisão a ser tomada, pelo engenheiro de processos, com relação a sua execução ou não no processo definido. Já um "Componente de Processo Abstrato" é aquele que não possui uma única forma de realização (admitindo variabilidades). Como ele necessita que outras decisões ainda sejam tomadas no momento da derivação de uma linha de processos em um "Processo Padrão" ou em um "Processo Definido para o Projeto", o mesmo não pode ser executado diretamente, até que todas as características tenham sido escolhidas e um componente concreto, que implemente esse componente abstrato, tenha sido selecionado (o que inclui a decisão em relação às opcionalidades existentes em sua arquitetura interna). Ambos os tipos de componentes de processo (concreto e abstrato) podem possuir uma arquitetura interna, que podem conter outros 
elementos de processo (componentes ou atividades). A Figura 1 apresenta alguns dos conceitos existentes na abordagem e como os mesmos estão inter-relacionados.

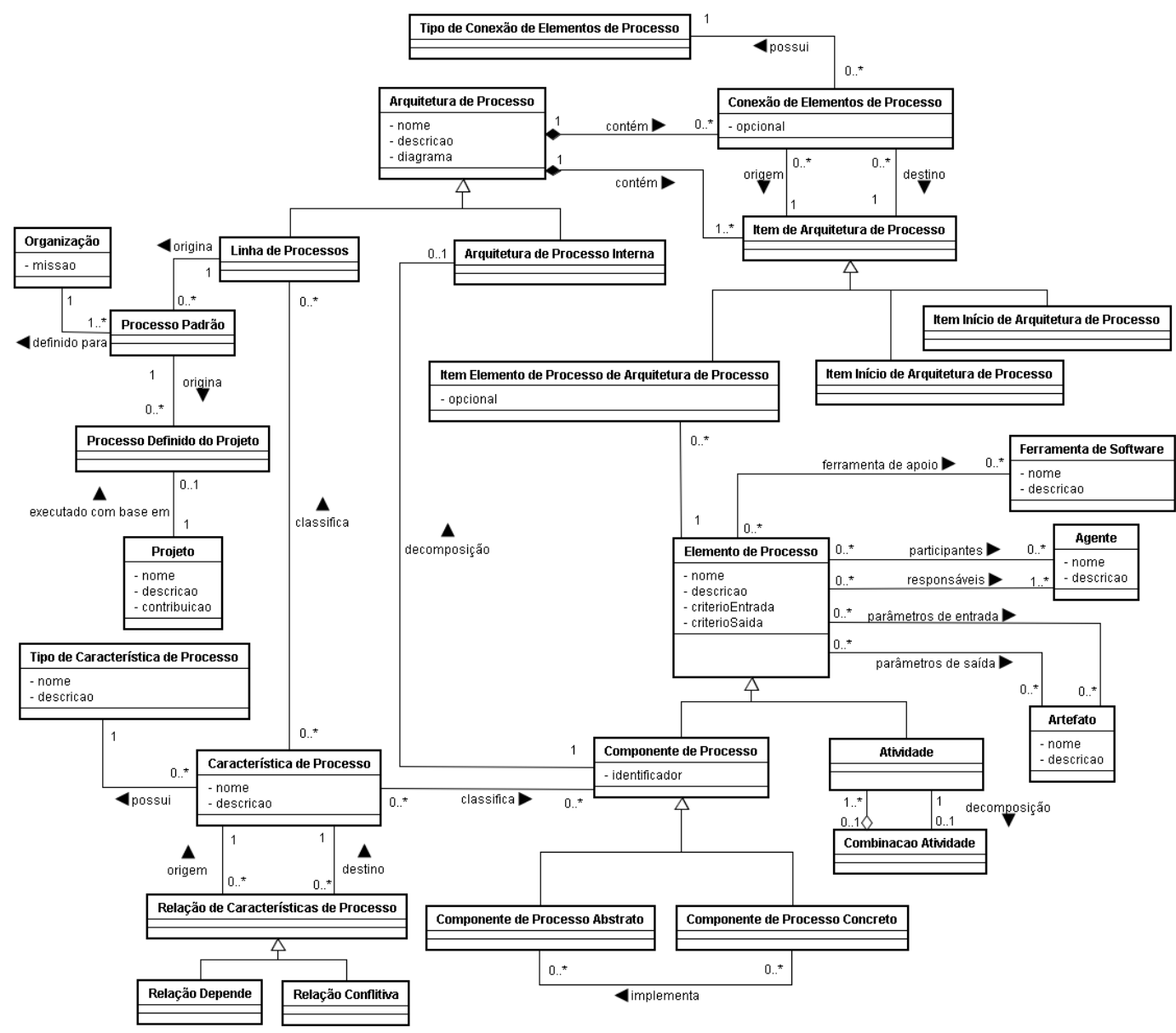

Figura 1. Modelo com os principais conceitos e seus relacionamentos adaptado de BARRETO [2011].

Uma "Arquitetura de Processo" é uma estrutura arquitetural que fornece a ordenação, as interfaces e as interdependências entre os "Elementos de Processo", sendo utilizada para o refinamento do processo para qualquer nível de detalhe. Essa ordenação é alcançada pela utilização do conceito de "Conexão de Elementos de Processo", representando o relacionamento entre dois elementos. Existem 5 (cinco) tipos de conexão entre elementos de processo, a saber: (i) Simples - conexão usada entre o item início da arquitetura e o primeiro elemento de processo e entre o último elemento de processo da arquitetura e o item fim da arquitetura; (ii) Fim-Início - conexão utilizada para representar que ao término da execução do elemento de processo origem, a execução do elemento destino pode ser iniciada; (iii) Início-Início - conexão utilizada para indicar que ao se iniciar a execução do elemento de processo origem, a execução do elemento de processo destino também deve ser iniciada; (iv) Fim-Fim - conexão utilizada para representar que ao término da execução do elemento de processo origem, a execução do elemento de processo destino também deve ser encerrada; (v) Início-Fim - conexão utilizada para representar que ao se iniciar a execução do elemento de processo origem, a execução do elemento de processo destino deve ser encerrada. 
Assim como um "componente de processo" essas conexões também podem ser opcionais.

Uma "Linha de Processos" é uma "arquitetura de processo" independente, ou seja, ela não faz parte da estrutura interna de um componente de processo. Em uma "Linha de Processos" a variabilidade é alcançada pelos pontos de variação, os quais são componentes que podem ser modificados ("Componentes de Processo Abstratos") de acordo com as "Características de Processo" selecionadas, como por exemplo, um "projeto com aquisição". As variantes de processo são os "componentes de processo concretos" candidatos e que são aplicados nesses pontos de variação.

Segundo BARRETO [2011] uma "Característica de Processo" pode ser vista como um aspecto, qualidade ou caracterização com a qual o processo precisa ser compatível. Dessa forma, tanto um "Componente de Processo" quanto uma "Linha de Processos" podem estar associados a mais de uma "Característica de Processo", possibilitando, assim, restringir a seleção e utilização desses elementos. Outro aspecto importante relacionado a "Características de Processo" é sua propriedade simétrica e transitiva, oriunda do tipo de relação entre as próprias características de processo, pois estas podem possuir uma relação de conflito ou de dependência umas com as outras.

\section{Abordagem Utilizada na Definição de Processos Reutilizáveis para Projetos com Aquisição}

$\mathrm{Na}$ abordagem para reutilização de processos proposta por BARRETO [2011], são apresentadas duas estratégias para definição de processos para reutilização, bottomup e top-down. A primeira é direcionada à definição de processos reutilizáveis a partir de Processos Legados (já existentes na organização). A segunda estratégia (top-down) é direcionada a organizações que desejam definir seus processos a partir do "zero", mas de forma que possam ser reutilizados em diferentes contextos e posteriormente evoluídos através da inclusão de novos elementos de processo.

Para a definição de processos reutilizáveis voltados a organizações que possuam projetos de desenvolvimento de software com aquisição, foi seguida a estratégia topdown, contudo, alguns passos da bottom-up foram realizados durante a execução do trabalho, com o objetivo de aproveitar o conhecimento contido nos ativos de processo da Instituição Implementadora COPPE/UFRJ. A top-down é composta por quatro passos principais: (i) Definir ou selecionar características de processo para a linha de processo; (ii) Definir ou selecionar e caracterizar os elementos de processo para a linha de processo; (iii) Estruturar e caracterizar a linha de processo; e (iv) Avaliar e aprovar a inclusão de itens reutilizáveis na biblioteca de processos reutilizáveis, conforme ilustrado na Figura 2. Tais passos não sendo necessariamente ordenados, uma vez que durante a execução de um passo pode-se perceber a necessidade de alguma definição adicional em um passo já realizado. 


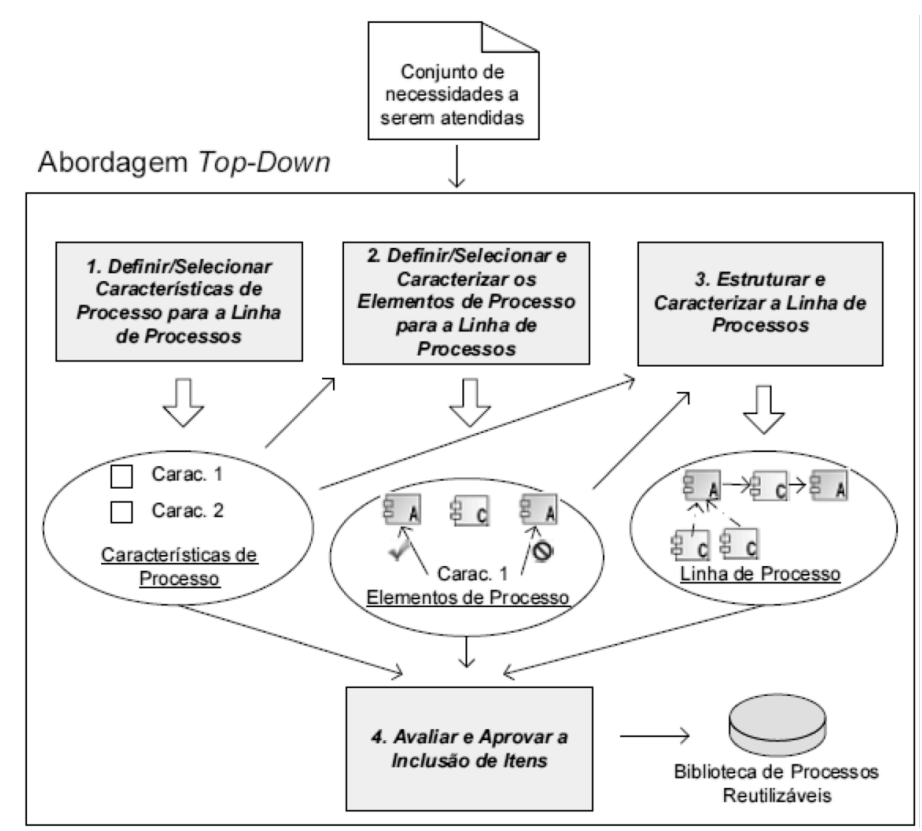

Figura 2. Abordagem Top-Down para definição de processos para reutilização [Barreto, 2011]

As subseções a seguir apresentam uma breve descrição do propósito de cada passo, contendo, ainda, como cada um foi executado para a definição de processos reutilizáveis voltados a organizações que possuem projetos de software com aquisição.

\subsection{Passo 1: Definição das Características para as Linhas de Processos}

O primeiro passo na abordagem é definir as características de processo. Tais características guiarão a definição ou seleção (nos casos em que já existam componentes ou linhas de processos disponíveis na base) dos demais elementos reutilizáveis. Para tal, é necessária a identificação do conjunto de necessidades que deverão ser atendidas pelos processos.

Para a execução do trabalho, o conjunto inicial de necessidades levou em consideração os processos e orientações que constam no Guia de Implementação do MR-MPS - Parte 8 [Softex, 2011], guia este voltado especificamente para organizações que adquirem software. Outras fontes de necessidades foram: (i) o modelo mais frequentemente utilizado pelas organizações públicas brasileiras em seus editais para a aquisição do desenvolvimento e/ou manutenção de sistemas, também conhecido como "contrato guarda-chuva" utilizando pontos de função; (ii) o fato de a organização adquirente poder realizar a aquisição com diferentes escopos, desde contratar apenas a especificação de requisitos até contratar todo o ciclo de vida do software; (iii) experiência vivenciada, durante os últimos 2 (dois) anos e meio, em projetos de desenvolvimento de software com aquisição na Eletrobras.

À medida que o trabalho de definição dos componentes e linhas de processos foi sendo realizado, novas necessidades foram sendo identificadas e adicionadas ao conjunto inicial. De posse dessas necessidades, foram derivados os tipos de características de processo (e as subsequentes características) aos quais as linhas e componentes de processo foram definidos e associados. Uma vez associadas a uma 
linha ou componente de processo, tais características possibilitam um meio de restringir a seleção e utilização desses elementos em uma posterior derivação para um processo padrão da organização ou um processo definido para um projeto. A seguir, é apresentado na Tabela 1 o resultado da derivação das origens das necessidades nas características de processos que serão utilizadas nos próximos passos:

\section{Tabela 1. Quadro de origem das necessidades que derivaram as características de processo}

\begin{tabular}{|c|c|c|c|}
\hline Origem da Necessidade & Necessidade & $\begin{array}{c}\text { Tipo da } \\
\text { Característica } \\
\end{array}$ & Característica de Processo \\
\hline \multirow[b]{2}{*}{ Objetivos do Trabalho } & \multirow{2}{*}{$\begin{array}{c}\text { Derivar processos aderentes aos } \\
\text { níveis iniciais de maturidade do } \\
\text { MR-MPS-SW }\end{array}$} & \multirow{2}{*}{$\begin{array}{l}\text { Compatibilidade } \\
\text { com Modelos e } \\
\text { Níveis de } \\
\text { Maturidade } \\
\end{array}$} & MR-MPS-SW - Nível G \\
\hline & & & MR-MPS-SW - Nível F \\
\hline \multirow[b]{2}{*}{ Objetivos do Trabalho } & \multirow{2}{*}{$\begin{array}{c}\text { Derivar processos capazes de } \\
\text { atender a organizações que } \\
\text { adquirem software }\end{array}$} & \multirow{2}{*}{$\begin{array}{c}\text { Tipo de } \\
\text { Desenvolvimento }\end{array}$} & Projeto com Aquisição \\
\hline & & & Projeto sem Aquisição \\
\hline \multirow{3}{*}{$\begin{array}{c}\text { GPR13 / GPR14 / } \\
\text { GPR17 - MR-MPS } \\
\text { [Softex, 2011] e } \\
\text { Experiência em projetos } \\
\text { vivenciados pelos } \\
\text { autores } \\
\end{array}$} & \multirow{3}{*}{$\begin{array}{l}\text { Derivar processos que } \\
\text { possibilitem a organização } \\
\text { adquirente acompanhar o } \\
\text { trabalho do fornecedor nas } \\
\text { diferentes fases do projeto }\end{array}$} & \multirow{3}{*}{$\begin{array}{c}\text { Acompanhament } \\
\text { o das atividades } \\
\text { do fornecedor }\end{array}$} & $\begin{array}{c}\text { Acompanha o levantamento de } \\
\text { requisitos }\end{array}$ \\
\hline & & & $\begin{array}{l}\text { Acompanha o projeto (design) da } \\
\text { solução }\end{array}$ \\
\hline & & & $\begin{array}{l}\text { Acompanha a construção do } \\
\text { produto }\end{array}$ \\
\hline \multirow{2}{*}{$\begin{array}{l}\text { Editais de Órgãos } \\
\text { Públicos }\end{array}$} & \multirow{2}{*}{$\begin{array}{l}\text { Derivar processos que apoiem } \\
\text { diferentes formas de } \\
\text { remuneração do fornecedor }\end{array}$} & \multirow{2}{*}{$\begin{array}{l}\text { Forma de } \\
\text { remuneração do } \\
\text { fornecedor }\end{array}$} & $\begin{array}{l}\text { Remuneração pela quantidade de } \\
\text { pontos de função }\end{array}$ \\
\hline & & & $\begin{array}{c}\text { Remuneração pela quantidade de } \\
\text { homens/hora empregados }\end{array}$ \\
\hline \multirow{4}{*}{$\begin{array}{c}\text { GPR2 - MR-MPS } \\
\text { [Softex, 2011] e Editais } \\
\text { de Órgãos Públicos }\end{array}$} & \multirow{4}{*}{$\begin{array}{l}\text { Derivar processos que utilizem } \\
\text { métodos apropriados para o } \\
\text { dimensionamento inicial de } \\
\text { tamanho e esforço do projeto }\end{array}$} & \multirow{4}{*}{$\begin{array}{l}\text { Forma de estimar } \\
\text { tamanho e } \\
\text { esforço do } \\
\text { projeto }\end{array}$} & $\begin{array}{l}\text { Estimativa utilizando contagem } \\
\text { indicativa de pontos de função }\end{array}$ \\
\hline & & & $\begin{array}{l}\text { Estimativa utilizando contagem } \\
\text { estimada de pontos de função }\end{array}$ \\
\hline & & & $\begin{array}{l}\text { Estimativa utilizando contagem } \\
\text { detalhada de pontos de função }\end{array}$ \\
\hline & & & $\begin{array}{c}\text { Estimativa utilizando base } \\
\text { histórica }\end{array}$ \\
\hline \multirow{3}{*}{$\begin{array}{l}\text { Objetivos do Trabalho e } \\
\text { Experiência em projetos } \\
\text { vivenciados pelos } \\
\text { autores }\end{array}$} & \multirow{3}{*}{$\begin{array}{l}\text { Derivar processos capazes de } \\
\text { atender a organizações que } \\
\text { adquirem algumas ou todas as } \\
\text { etapas do desenvolvimento do } \\
\text { software }\end{array}$} & \multirow{3}{*}{$\begin{array}{l}\text { Escopo de } \\
\text { aquisição }\end{array}$} & $\begin{array}{c}\text { Aquisição somente do } \\
\text { levantamento e definição dos } \\
\text { requisitos }\end{array}$ \\
\hline & & & $\begin{array}{c}\text { Aquisição de todo o ciclo de } \\
\text { vida de desenvolvimento do } \\
\text { software }\end{array}$ \\
\hline & & & $\begin{array}{c}\text { Aquisição a partir do } \\
\text { levantamento dos requisitos }\end{array}$ \\
\hline \multirow{2}{*}{$\begin{array}{c}\text { MED1 - MR-MPS } \\
\text { [Softex, 2011] e [Rocha } \\
\text { et al., 2012] }\end{array}$} & \multirow{2}{*}{$\begin{array}{l}\text { Derivar processos de medição } \\
\text { capazes de apoiar a definição de } \\
\text { medidas ou indicadores } \\
\text { alinhados aos objetivos de } \\
\text { negócio da organização }\end{array}$} & \multirow{2}{*}{$\begin{array}{l}\text { Método utilizado } \\
\text { para apoiar a } \\
\text { definição de } \\
\text { medidas }\end{array}$} & $\begin{array}{l}\text { Definir medidas utilizando o } \\
\text { método GQM }\end{array}$ \\
\hline & & & $\begin{array}{l}\text { Definir medidas utilizando o } \\
\text { método GQ(I)M }\end{array}$ \\
\hline \multirow{2}{*}{$\begin{array}{c}\text { MED7 - MR-MPS } \\
\text { [Softex, 2011] e [Rocha } \\
\text { et al., 2012] }\end{array}$} & \multirow{2}{*}{$\begin{array}{l}\text { Derivar processos de medição } \\
\text { que apoiem diferentes formas de } \\
\text { divulgação de seus resultados }\end{array}$} & \multirow{2}{*}{$\begin{array}{c}\text { Forma utilizada } \\
\text { para divulgação } \\
\text { dos resultados de } \\
\text { medição }\end{array}$} & $\begin{array}{l}\text { Reportar resultados de medição } \\
\text { por e-mail }\end{array}$ \\
\hline & & & $\begin{array}{l}\text { Reportar resultados de medição } \\
\text { através de reunião }\end{array}$ \\
\hline \multirow{3}{*}{$\begin{array}{l}\text { [Vargas, 2010], [Costa, } \\
\text { 2011] e [PMI, 2008] }\end{array}$} & \multirow{3}{*}{$\begin{array}{l}\text { Derivar processos para gerência } \\
\text { de portfólio que utilizem } \\
\text { diferentes métodos para seleção } \\
\text { de projetos para a formação do } \\
\text { portfólio }\end{array}$} & \multirow{3}{*}{$\begin{array}{l}\text { Método para } \\
\text { seleção de } \\
\text { projetos para } \\
\text { compor portfólio }\end{array}$} & $\begin{array}{c}\text { Selecionar portfólio utilizando } \\
\text { MTP }\end{array}$ \\
\hline & & & $\begin{array}{c}\text { Selecionar projetos utilizando } \\
\text { AHP } \\
\end{array}$ \\
\hline & & & $\begin{array}{l}\text { Selecionar projetos utilizando } \\
\text { abordagem de mapeamento }\end{array}$ \\
\hline
\end{tabular}

Os tipos de características "Compatibilidade com Modelos e Níveis de Maturidade" e "Escopo de aquisição" resultaram em características fortemente voltadas 
à caracterização de linhas de processos diferentes, cabendo a essa última uma explicação mais detalhada de seu propósito:

- Aquisição somente do levantamento e definição dos requisitos do produto: essa característica está relacionada a linhas de processos ou componentes que apoiem projetos com aquisição somente das atividades relacionadas ao levantamento e definição dos requisitos do produto. Projetos desse tipo finalizam com a aprovação da definição dos requisitos pela organização adquirente.

- Aquisição de todo o ciclo de vida de desenvolvimento do software: essa característica está relacionada a linhas de processos ou componentes que suportem projetos com aquisição de todas as atividades relacionadas ao desenvolvimento de um produto, do levantamento de requisitos até sua implantação em ambiente de produção.

- Aquisição a partir do levantamento dos requisitos: essa característica está relacionada a linhas de processos ou componentes que apoiem projetos com aquisição das atividades a serem realizadas após a definição dos requisitos, que podem ou não terem sido definidos internamente. Projetos desse tipo tem início com a contratada recebendo uma lista de requisitos e termina com a implantação do produto.

Ao final, foi determinado, para cada característica, qual o seu tipo de relacionamento com as demais, conforme os tipos de relação entre características existentes na abordagem. Como resultado desse mapeamento, a Tabela 2 foi construída de forma a facilitar a consulta durante a definição dos componentes e linhas de processos, como também, no posterior processo de avaliação do trabalho.

Tabela 2. Quadro demonstrativo das relações existentes entre as características de processo definidas

\begin{tabular}{|c|c|c|c|c|c|c|c|c|c|c|c|c|c|c|c|c|}
\hline & Característica de Processo & \begin{tabular}{l|l}
1 & 2 \\
$r$
\end{tabular} & 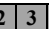 & 4 & \begin{tabular}{l|l|l}
5 & 6
\end{tabular} & \begin{tabular}{|l|l|l|r|r|r|r|r|r|}
7 & 8 \\
\end{tabular} & 9 & \begin{tabular}{|l|l|}
10 & 11 \\
\end{tabular} & $12 \mid 13$ & \begin{tabular}{l|l|l}
3 & 14 & 1 \\
\end{tabular} & \begin{tabular}{l|l|l|}
15 & 16 & \\
\end{tabular} & \begin{tabular}{l|l|l|l|l}
6 & 17 & 1 \\
\end{tabular} & \begin{tabular}{l|l|l|}
18 & 19 & 19 \\
\end{tabular} & & & 223 \\
\hline 1 & MR-MPS - Nível G & & & & & & & & & & & & & & & \\
\hline 2 & MR-MPS - Nível F & & & & & & & & & & & & & & & \\
\hline 3 & Projeto com Aquisição & & & C & & & & & & & & & & & & \\
\hline 4 & Projeto sem Aquisição & & C & & & & & & & 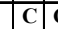 & \begin{tabular}{l|l|}
$\mathbf{C}$ & $\mathbf{C}$ \\
\end{tabular} & & & & & \\
\hline 5 & Acompanha o levantamento de requisitos & & & & & & & & & & & & & & & \\
\hline 6 & Acompanha o projeto (design) da solução & & & & & & & & & & & & & & & \\
\hline 7 & Acompanha a construção do produto & & & & & & & & & & & & & & & \\
\hline 8 & Remuneração pela quantidade de pontos de função & & & & & & C & & & & & & & & & \\
\hline 9 & Remuneração pela quantidade de homens/hora empregados & & & & & C & & & & & & & & & & \\
\hline 10 & Estimativa utilizando contagem indicativa de pontos de função & & & & & & & C & \begin{tabular}{l|l}
$\mathbf{C}$ & $\mathbf{C}$ \\
\end{tabular} & & & & & & & \\
\hline 11 & Estimativa utilizando contagem estimada de pontos de função & & & & & & & 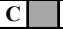 & \begin{tabular}{|l|l|lll}
$\mathbf{C}$ & $\mathbf{C}$ \\
\end{tabular} & & & & & & & \\
\hline 12 & Estimativa utilizando contagem detalhada de pontos de função & & & & & & & \begin{tabular}{l|l|l}
$\mathbf{C}$ & $\mathbf{C}$ & \\
\end{tabular} & \begin{tabular}{|c|c|}
$\mathbf{C}$ \\
\end{tabular} & \begin{tabular}{l|llll} 
& $\mathbf{C}$ & $(1$ \\
\end{tabular} & C & & & & & \\
\hline 13 & Estimativa utilizando base histórica & & & & & & & \begin{tabular}{l|l|l}
$\mathbf{C}$ & $\mathbf{C}$ \\
\end{tabular} & \begin{tabular}{|c|c|c|} 
C & \\
\end{tabular} & & & & & & & \\
\hline 14 & Aquisição somente do levantamento e definição dos requisitos & & D & C & & & & & C & ( & \begin{tabular}{l|l|}
$\mathbf{C}$ & $\mathbf{C}$ \\
\end{tabular} & & & & & \\
\hline 15 & Aquisição de todo o ciclo de vida de desenvolvimento do software & & D & $\mathbf{C}$ & & & & & C| & C & $\mathbf{C}$ & & & & & \\
\hline 16 & Aquisição a partir do levantamento dos requisitos & & D & C & & & & & & 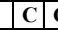 & \begin{tabular}{l|l}
$\mathbf{C}$ & \\
\end{tabular} & & & & & \\
\hline 17 & Definir medidas utilizando o método GQM & & & & & & & & & & & & C & & & \\
\hline 18 & Definir medidas utilizando o método GQ(I)M & & & & & & & & & & & 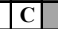 & & & & \\
\hline 19 & Reportar resultados de medição por e-mail & & & & & & & & & & & & & C & & \\
\hline 20 & Reportar resultados de medição através de reunião & & & & & & & & & & & & C & & & \\
\hline 21 & Selecionar portfólio utilizando MTP & & & & & & & & & & & & & & C & \begin{tabular}{c|c|c|c|c}
$C$ & $\mathbf{C}$ \\
\end{tabular} \\
\hline 22 & Selecionar projetos utilizando AHP & & & & & & & & & & & & & & 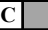 & $\mathbf{C}$ \\
\hline 23 & Selecionar projetos utilizando abordagem de mapeamento & & & & & & & & & & & & & & $\mathbf{C}$ & C] \\
\hline
\end{tabular}




\subsection{Passo 2: Definição e Caracterização dos Elementos de Processo}

O próximo passo refere-se à construção (definição) dos componentes de processo necessários à composição das linhas de processos de software. Esse passo da abordagem possui as seguintes atividades: (i) definir ou selecionar (caso existam componentes disponíveis na Biblioteca, oriundos de definições anteriores) os elementos de processo que serão usados para atender as características que foram definidas; (ii) identificar semelhanças e variabilidades nesses elementos de processo, ou seja, que partes serão comuns (componentes concretos) para todos os processos derivados a partir da linha e quais poderão variar (componentes abstratos); (iii) à medida que os componentes forem sendo definidos, devem ser caracterizados de acordo com as características de processo definidas no passo 1 (um).

As informações contidas nos elementos de processo definidos referentes a ferramentas, artefatos, responsáveis e participantes, bem como as orientações contidas na descrição de cada um, tinham como objetivo representar sugestões de boas práticas retiradas da literatura e dos modelos de maturidade em processos, voltadas para organizações que possuam projetos de desenvolvimento de software com aquisição e que ainda não iniciaram o seu processo de melhoria em processos ou estão em um estágio inicial. Portanto, tais elementos de processos foram projetados e definidos para serem diretamente reutilizados por essas organizações, sem a necessidade de grandes adaptações. Entretanto, não fazia parte do trabalho prover templates para cada um desses artefatos, ou sugestão de ferramentas específicas, ficando, tais atividades, a cargo de cada organização. Porém, a maioria dos elementos de processo definidos possuem em sua descrição alguns dos pontos que precisam constar de tais artefatos ou ferramentas.

Para facilitar a definição dos componentes e atividades utilizou-se o template para definição de processos do grupo de Qualidade de Software da COPPE/UFRJ, acrescido de algumas informações necessárias à abordagem utilizada. Para atender a necessidade de derivar processos que utilizem métodos apropriados para o dimensionamento inicial de tamanho e esforço do projeto, foi definido um componente abstrato "Dimensionar o tamanho do projeto" contendo quatro variantes, possibilitando a derivação de um processo definido para um projeto que adote um método apropriado de estimativa, mediante as informações já disponíveis sobre os requisitos do produto. A Tabela 3, abaixo, demonstra uma dessas variantes:

\section{Tabela 3. Variante do Componente de Processo "Dimensionar o tamanho do projeto"}

\begin{tabular}{|l|l|}
\hline Dimensionar o tamanho do projeto através da contagem indicativa de pontos de função \\
\hline Identificador: & COP.GPR.PLA.ABS.0002 \\
\hline Nome: & Dimensionar o tamanho do projeto através da contagem indicativa de pontos de função \\
\hline Tipo: & Abstrato \\
\hline Descrição: & $\begin{array}{l}\text { Dimensionar o tamanho funcional do produto baseado na lista de requisitos macro, } \\
\text { aplicando o método de contagem indicativa de pontos de função, conforme definido } \\
\text { pela NESMA (Netherlands Software Metrics Association). A execução deste } \\
\text { componente é realizada pelo adquirente e seu resultado deve ser ratificado junto ao } \\
\text { fornecedor. }\end{array}$ \\
\hline Definido por: & COPPE/UFRJ \\
\hline Critérios de Entrada: & Ter-se a lista de requisitos macro para o projeto. \\
\hline Critérios de Saída: & $\begin{array}{l}\text { Ter-se o tamanho do projeto dimensionado através de uma indicação do tamanho } \\
\text { funcional para o produto. }\end{array}$ \\
\hline Responsável: & Analista de Requisitos \\
\hline
\end{tabular}




\begin{tabular}{|l|l|l|}
\hline Participantes: & Fornecedor de Requisitos; Fornecedor. \\
\hline Ferramentas de Apoio: & Planilha Eletrônica; Processador de textos. \\
\hline Artefatos Requeridos: & $\begin{array}{l}\text { Requisitos identificados de forma macro (lista de requisitos); Template da Planilha de } \\
\text { Contagem de Pontos de Função; Termo de Abertura do Projeto. }\end{array}$ \\
\hline Artefatos Produzidos: & $\begin{array}{l}\text { Planilha de Contagem de Pontos de Função preenchida com uma indicação de tamanho } \\
\text { funcional do produto }\end{array}$ \\
\hline $\begin{array}{l}\text { Características } \\
\text { Atendidas: }\end{array}$ & $\begin{array}{l}\text { MR-MPS-SW - Nível G; MR-MPS-SW - Nível F; Projeto com Aquisição; Estimativa } \\
\text { utilizando contagem indicativa de pontos de função. }\end{array}$ \\
\hline Medidas: & ESF_DIM_TAM - Esforço para realizar o dimensionamento de tamanho do projeto \\
\hline $\begin{array}{l}\text { Variantes deste } \\
\text { componente: }\end{array}$ & - & \\
\hline Arquitetura Interna: &
\end{tabular}

As medidas propostas em cada componente de processo foram definidas com o objetivo de possibilitar a análise de desempenho e capacidade do processo. Contudo, essas medidas não esgotam esse propósito. Dessa forma, as organizações podem optar pelo seu uso ou não, substituindo-as por outras direcionadas ao alcance de seus objetivos de medição. Tais medidas foram propostas com base em ROCHA et al. [2012], onde maiores informações sobre tais medidas podem ser encontradas, além da sugestão de outras que podem ser adicionadas aos componentes.

\subsection{Passo 3: Estruturação e Caracterização das Linhas de Processos}

Após a definição dos componentes de processo e das atividades necessárias ao atendimento do escopo pretendido para a linha de processos, é realizada a sua estruturação seguindo os seguintes passos: (i) escolher quais componentes serão opcionais na linha de processos; (ii) estabelecer as conexões entre os elementos de processo; (iii) determinar se a conexão incluída é obrigatória ou opcional; (iv) mapear características de processo diretamente para a linha de processos.

Como o objetivo do trabalho era a definição de componentes e linhas de processos que atendessem aos níveis G e F do MR-MPS-SW e que possibilitassem a derivação de processos capazes de atender a organizações que adquirem algumas ou todas as etapas do desenvolvimento do software, foi necessária a criação de 10 (dez) linhas de processos, subdivididas em dois propósitos de uso.

- Linhas de processos voltadas à utilização em projetos: (i) Linha de processos para aquisição somente do levantamento e definição dos requisitos do produto Nível G; (ii) Linha de processos para aquisição de todo o ciclo de vida de desenvolvimento do software - Nível G; (iii) Linha de processos para aquisição a partir do levantamento dos requisitos - Nível G; (iv) Linha de processos para aquisição somente do levantamento e definição dos requisitos do produto - Nível F; 
(v) Linha de processos para aquisição de todo o ciclo de vida de desenvolvimento do software-Nível F; (vi) Linha de processos para aquisição a partir do levantamento dos requisitos - Nível F

- Linhas de processos para o contexto organizacional: (i) Linha de processos para Garantia da Qualidade-GQA; (ii) Linha de processos para Gerência de Configuração - GCO; (iii) Linha de processos para Medição - MED; (iv) Linha de processos para Gerência de Portfólio de Projetos - GPP

A Figura 3 apresenta o resultado da estruturação de uma das linhas de processos, citadas anteriormente.

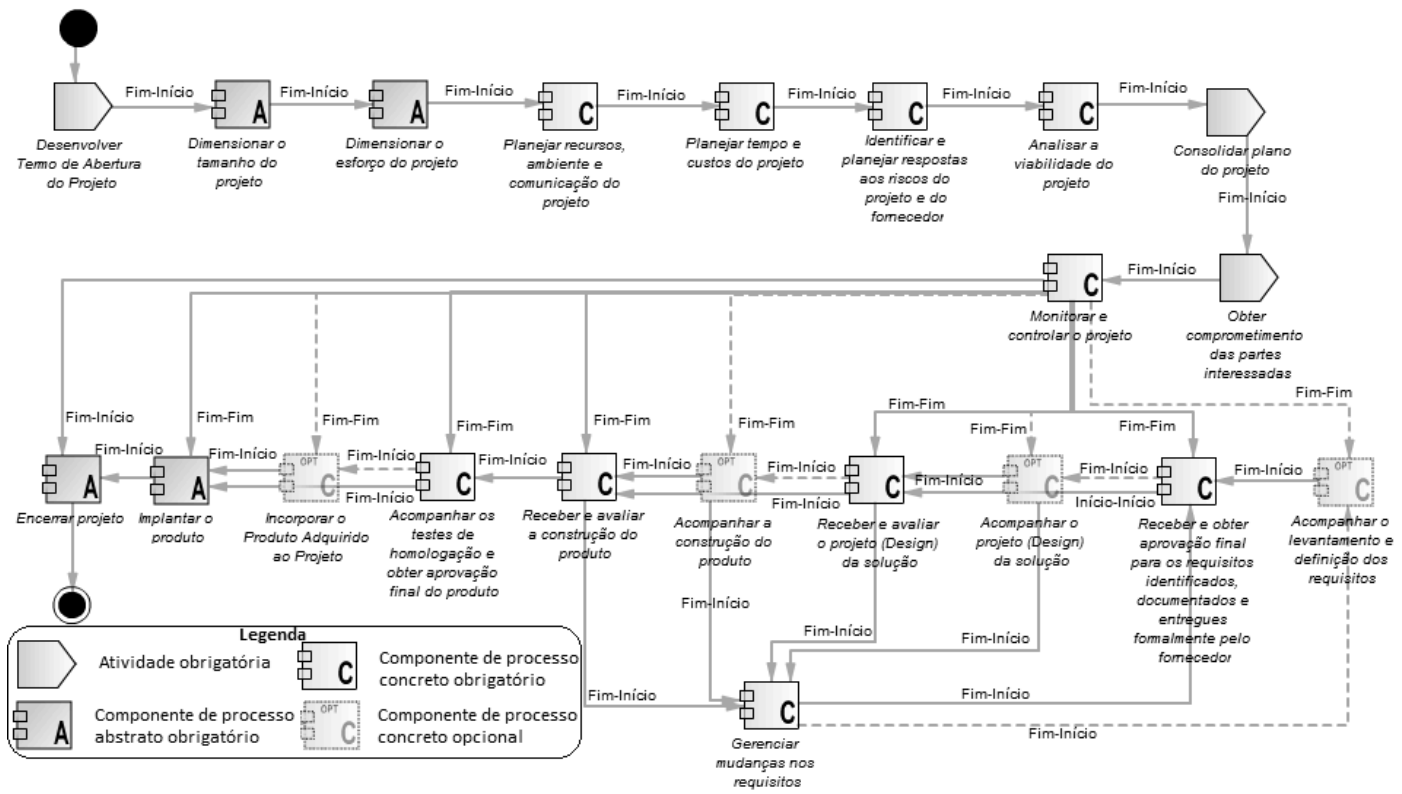

Figura 3. Arquitetura da linha de processos para projetos com aquisição de todo o ciclo de vida de desenvolvimento do software - Nível G [Cardoso, 2012]

Para atender a todos os processos e resultados esperados no nível $\mathrm{F}$ do MRMPS-SW, a organização precisa implementar o processo Aquisição (AQU). Tal processo também foi definido sob a forma de componentes e linhas de processos no trabalho de NUNES [2011], podendo ser reutilizado para alcançar esse objetivo.

\subsection{Passo 4: Avaliação dos Itens Reutilizáveis}

Esse último passo tem como objetivo avaliar se a linha de processos definida e seus elementos são adequados para atender as necessidades estabelecidas. Além disso, verificar se a abordagem para definição de processos reutilizáveis foi aplicada corretamente. Portanto, esse é um passo de suma importância, pois garante a qualidade do trabalho que foi desenvolvido.

Durante a execução do trabalho, a avaliação foi feita com dois enfoques: (i) quanto ao formato das linhas e componentes de processo, ou seja, se foram preenchidos todos os campos necessários à definição de tais elementos; (ii) quanto ao conteúdo das linhas e componentes processo, ou seja, se o conteúdo daquele elemento está de acordo com o propósito pelo qual ele foi definido. Dessa forma, foram utilizados 4 (quatro) laudos de avaliação (no formato de checklists), sendo um para cada foco de avaliação: 
(i) Forma do Componente de Processo; (ii) Conteúdo do Componente de Processo; (iii) Forma da Linha de Processos; (iv) Conteúdo da Linha de Processos.

Para condução do trabalho de revisão foi convidada uma especialista com experiência em projetos com aquisição, definição de processos e em reutilização de componentes. Nessa revisão por pares, foi verificado se os componentes utilizados, o conjunto de características de processo definidas, o sequenciamento lógico entre os componentes, os pontos de variação e variantes foram definidos de acordo com os conceitos de reutilização de processos definidos em BARRETO [2011] e se atendiam aos objetivos e cenários de projetos com aquisição propostos.

Após a finalização da definição de cada linha de processos, a linha e todos os elementos novos eram enviados ao revisor que utilizava o laudo de avaliação como checklist para condução do trabalho e realizava os apontamentos das não conformidades encontradas, dúvidas ou sugestões de melhoria diretamente no texto, utilizando o mecanismo de comentários existente no processador de textos. Ao término de cada avaliação, onde todas as dúvidas do revisor já haviam sido sanadas, o documento era devolvido e as não conformidades e sugestões de melhoria contabilizadas e corrigidas/ajustadas. Como pode ser observado na Tabela 4 , durante a primeira rodada de revisão, muitas dúvidas surgiram e as principais não conformidades encontradas foram na forma como os dados básicos dos componentes abstratos foram preenchidos (pois nos casos em que o componente é abstrato e possui variantes decidiu-se que os critérios de entrada, saída, responsável, participantes, ferramentas, artefatos requeridos e artefatos produzidos, não deveriam ser preenchidos) e na consistência entre os dados básicos dos componentes de processo concretos com suas as atividades presentes na arquitetura interna.

Tabela 4. Apuração de não conformidades por critério de avaliação encontradas durante a revisão por pares para as linhas de processo referentes ao nível G

\begin{tabular}{|c|c|c|c|c|c|c|c|c|c|c|c|c|c|c|}
\hline \multirow{2}{*}{$\begin{array}{l}\text { Laudo de } \\
\text { Avaliação }\end{array}$} & \multirow{2}{*}{ Critério } & \multirow{2}{*}{$\begin{array}{c}\text { Qtd. de } \\
\text { itens de } \\
\text { avaliação } \\
\text { por } \\
\text { critério }\end{array}$} & \multicolumn{4}{|c|}{ Só Requisitos - Nível G } & \multicolumn{4}{|c|}{ Todo Ciclo de Vida - Nível G } & \multicolumn{4}{|c|}{ Após Requisitos - Nível G } \\
\hline & & & \begin{tabular}{|c|} 
Qtd. \\
Elem. \\
Avl. \\
\end{tabular} & \begin{tabular}{|c|} 
Qtd. \\
Elm x \\
Crit.
\end{tabular} & \begin{tabular}{|c|} 
Não \\
Conf.
\end{tabular} & \begin{tabular}{|c|}
$\%$ de \\
Não \\
Conf.
\end{tabular} & $\begin{array}{l}\text { Qtd. } \\
\text { Elem. } \\
\text { Av. }\end{array}$ & \begin{tabular}{|c|} 
Qtd. \\
Em x \\
Crit. \\
\end{tabular} & $\begin{array}{c}\text { Não } \\
\text { Conf. }\end{array}$ & \begin{tabular}{|c|}
$\%$ de \\
Não \\
Conf.
\end{tabular} & \begin{tabular}{|c|} 
Qtd. \\
Elem. \\
Avl. \\
\end{tabular} & \begin{tabular}{|c|} 
Qtd. \\
Em x \\
Crit. \\
\end{tabular} & \begin{tabular}{|c} 
Não \\
Conf.
\end{tabular} & \begin{tabular}{|c}
$\%$ de \\
Não \\
Conf.
\end{tabular} \\
\hline \multirow{3}{*}{$\begin{array}{c}\text { Linha de } \\
\text { Processo - } \\
\text { Forma } \\
\end{array}$} & Dados Básicos da Linha de Processos & 5 & 1 & 5 & - & 0 & 1 & 5 & - & 0 & \begin{tabular}{|l|l}
1 &
\end{tabular} & 5 & - & 0 \\
\hline & Características de Processo da Linha de Processos & 3 & 1 & 3 & & 0 & 1 & 3 & - & 0 & 1 & 3 & & 0 \\
\hline & Arquitetura da Linha de Processos & 6 & 1 & 6 & - & 0 & 1 & 6 & - & 0 & 1 & 6 & - & 0 \\
\hline \multirow{4}{*}{$\begin{array}{c}\text { Linha de } \\
\text { Processo - } \\
\text { Conteúdo } \\
\end{array}$} & Dados Básicos da Linha de Processos & 2 & 1 & 2 & - & 0 & 1 & 2 & - & 0 & 1 & 2 & - & 0 \\
\hline & Características de Processo da Linha de Processos & 9 & 1 & 9 & - & 0 & 1 & 9 & - & 0 & 1 & 9 & - & 0 \\
\hline & Componentes de Processo da Linha de Processos & 7 & 1 & 7 & - & 0 & 1 & 7 & - & 0 & 1 & 7 & - & 0 \\
\hline & \begin{tabular}{|l|} 
Arquitetura da Linha de Processos \\
\end{tabular} & 7 & 1 & 7 & & 0 & 1 & 7 & - & 0 & 1 & 7 & - & 0 \\
\hline \multirow{6}{*}{$\begin{array}{c}\text { Componente } \\
\text { de Processo } \\
\text { Forma } \\
\end{array}$} & Relevância do Componente de Processo & 1 & 81 & 81 & & 0 & 35 & 35 & - & 0 & 12 & 12 & & 0 \\
\hline & \begin{tabular}{|l|} 
Dados Básicos do Componente de Processo \\
\end{tabular} & 17 & 81 & 1.377 & 14 & 1,017 & 35 & 595 & - & 0 & 12 & 204 & & 0 \\
\hline & Características de Processo do Componente de Processo & 3 & 81 & 243 & - & 0 & 35 & 105 & - & 0 & 12 & 36 & - & 0 \\
\hline & Variação do Componente de Processo & 4 & 81 & 324 & - & 0 & 35 & 140 & - & 0 & 12 & 48 & - & 0 \\
\hline & Arquitetura Interna do Componente de Processo & 6 & 81 & 486 & - & 0 & 35 & 210 & - & 0 & 12 & 72 & - & 0 \\
\hline & Medidas do Componente de Processo & 2 & 81 & 162 & - & 0 & 35 & 70 & - & 0 & 12 & 24 & - & 0 \\
\hline \multirow{6}{*}{$\begin{array}{c}\text { Componente } \\
\text { de Processo } \\
\text { Conteúdo } \\
\end{array}$} & Relevância do Componente de Processo & 1 & 81 & 81 & - & 0 & 35 & 35 & - & 0 & 12 & 12 & - & 0 \\
\hline & \begin{tabular}{|l|} 
Dados Básicos do Componente de Processo \\
\end{tabular} & 11 & 81 & 891 & 39 & 4,377 & 35 & 385 & 20 & 5,195 & 12 & 132 & & 0 \\
\hline & Características de Processo do Componente de Processo & 5 & 81 & 405 & - & 0 & 35 & 175 & - & 0 & 12 & 60 & & 0 \\
\hline & Variação do Componente de Processo & 4 & 81 & 324 & & 0 & 35 & 140 & & 0 & 12 & 48 & & 0 \\
\hline & Arquitetura Interna do Componente de Processo & 5 & 81 & 405 & 3 & 0,741 & 35 & 175 & & 0 & 12 & 60 & & 0 \\
\hline & Medidas do Componente de Processo & 3 & 81 & 243 & - & 0 & 35 & 105 & - & 0 & 12 & 36 & $=$ & 0 \\
\hline \multirow{6}{*}{ Geral } & \multicolumn{2}{|l|}{ Questionamentos do Revisor } & \multicolumn{4}{|c|}{6} & \multicolumn{4}{|c|}{6} & \multicolumn{4}{|c|}{0} \\
\hline & \multicolumn{2}{|l|}{ 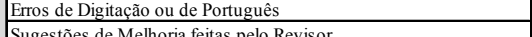 } & \multicolumn{4}{|c|}{$\frac{5}{5}$} & \multicolumn{4}{|c|}{$\frac{1}{2}$} & \multicolumn{4}{|c|}{0} \\
\hline & \multicolumn{2}{|l|}{ Sugestões de Melhoria feitas pelo Revisor } & \multirow{2}{*}{\multicolumn{4}{|c|}{$\frac{5}{19}$}} & \multirow{2}{*}{\multicolumn{4}{|c|}{$\frac{2}{8}$}} & \multirow{2}{*}{\multicolumn{4}{|c|}{$\frac{3}{2}$}} \\
\hline & \multirow{2}{*}{\multicolumn{2}{|c|}{$\begin{array}{r}\text { Qtd de Componentes Novos } \\
\text { Qtd de Atividades Novas }\end{array}$}} & & & & & & & & & & & & \\
\hline & & & \multirow{2}{*}{\multicolumn{4}{|c|}{62}} & \multirow{2}{*}{\multicolumn{4}{|c|}{27}} & \multicolumn{4}{|c|}{10} \\
\hline & \multicolumn{2}{|c|}{$\begin{array}{r}\text { Total: } \\
\text { Q }\end{array}$} & & & & & & & 35 & & & & & \\
\hline
\end{tabular}


Sugestões de melhoria foram quase sempre identificadas e acatadas, elas versavam, principalmente, sobre o uso de um nome mais adequado para um determinado artefato ou sobre uma melhor forma para descrever determinada atividade, tornando mais claro o seu propósito.

\section{Considerações Finais}

Durante a execução do trabalho e mediante a revisão bibliográfica feita, algumas sugestões poderiam ser adicionadas aos comentários já existentes no Guia de Implementação do MR-MPS - Parte 8, sugestões essas que foram implementadas nos componentes de processo definidos, tais como: (i) A execução das atividades de planejamento do projeto em conjunto (adquirente e fornecedor); (ii) Levar em consideração os fatores apontados na revisão da literatura como riscos, barreiras e problemas, na análise e monitoramento dos riscos do projeto; (iii) $\mathrm{O}$ compartilhamento entre adquirente e fornecedor de todos os artefatos gerados durante o planejamento e execução do projeto; (iv) Utilizar profissionais da equipe de TI da organização adquirente para acompanhar o trabalho desempenhado pelo fornecedor durante todo o ciclo de vida do projeto, registrando as não conformidades encontradas e mantendo o gerente do projeto da organização adquirente sempre informado sobre andamento e status do projeto; (v) Reunir os principais interessados no início do projeto (reunião de kick-off), juntamente com os representantes da empresa contratada e da TI da empresa contratante, explicando as cláusulas contratuais, bem como, as obrigações e direitos de cada um dos envolvidos; (vi) Registrar todos os problemas, tanto os que tiveram origem na organização adquirente, quanto no fornecedor, na base histórica de projetos, bem como qual foi a solução empregada.

No tocante aos órgãos públicos brasileiros, acredita-se que o fruto deste trabalho possa ser de grande utilidade a tais organizações, pois segundo o próprio TCU [2010] o ente público precisará garantir que qualquer fornecedor contratado entregue todos os resultados esperados que caracterizam o nível de capacidade pretendido, como parte da melhoria de seu processo. Mesmo o ente público que não tem interesse em avaliação oficial de seu processo está obrigado a exigir serviços que tenham qualidade, o que pressupõe a adoção de processos internos de software com maturidade suficiente para manter tais contratos. Dessa forma, o "Entendimento V" da Nota Técnica SEFTI/TCU $\mathrm{n}^{\mathrm{o}} 05$ estabelece a possibilidade de inclusão, na especificação técnica dos serviços a serem realizados, todos os resultados esperados que, segundo modelos de qualidade de processo aderentes à norma ABNT NBR ISO/IEC 15.504, tais como CMMI ou MPS.BR, caracterizam um dado nível de capacidade de processo de software, desde que tal nível reflita as escolhas estratégicas da organização para o seu processo de software e a sua real capacidade de avaliar tecnicamente os artefatos e produtos entregues [TCU, 2010].

Como trabalho futuro, pretende-se utilizar tais linhas de processos em projetos reais. Para tal, a Eletrobras já demonstrou interesse e pretende utilizá-las para a definição de seus processos, adaptando algumas atividades à sua realidade e ferramentas já utilizadas. Tal aplicação resultaria em uma evolução e continuidade do trabalho, pois demandaria, com o tempo, a definição de componentes de processo para os demais níveis de maturidade do MR-MPS, além de outros voltados a cenários de aquisição diferentes, tais como, a aquisição somente de código ou aquisição somente de testes em um projeto. A linha de processos para gerência de portfólio de projetos também poderia 
ser evoluída, através da inclusão de novos componentes em sua arquitetura e novas variantes para a seleção de projetos.

Também como trabalho futuro, as linhas de processos definidas no trabalho poderiam ser unificadas em uma linha de processos somente, possibilitando a derivação das demais através da seleção de suas características.

\section{Agradecimentos}

O desenvolvimento desse trabalho contou com o apoio e experiência de diversos profissionais aos quais agradecemos. São eles: Ahilton Silva Barreto, Elaine Nunes, Gleison Santos e Rômulo Coutinho. Agradecemos, também, a ELETROBRAS pelo apoio e interesse na aplicação dos resultados oriundos desse trabalho.

\section{Referências}

Barreto, A.S. (2011) "Uma Abordagem para Definição de Processos Baseada em Reutilização Visando à Alta Maturidade em Processos", Tese de D.Sc., Programa de Pós-graduação em Engenharia de Sistemas e Computação - PESC/COPPE, Universidade Federal do Rio de Janeiro - UFRJ, Rio de Janeiro.

Cardoso, F.S. (2012) "Definição de Processos Reutilizáveis para Projetos com Aquisição", Dissertação de M.Sc., Programa de Pós-graduação em Engenharia de Sistemas e Computação - PESC/COPPE, Universidade Federal do Rio de Janeiro UFRJ Rio de Janeiro.

Costa, H.R. (2011) "Apoio à Seleção de Portfólio de Projetos de Software Baseado na Moderna Teoria do Portfólio", Tese de D.Sc., Programa de Pós-graduação em Engenharia de Sistemas e Computação - PESC/COPPE, Universidade Federal do Rio de Janeiro - UFRJ, Rio de Janeiro.

Khan, S.U., Niazi, M., Ahmad, R. (2009) "Critical barriers for offshore software development outsourcing vendors: A systematic literature review". In: 16th AsiaPacific Software Engineering Conference, APSEC 2009, December 1, 2009 December 3, 2009, pp. 79-86, Penang, Malaysia.

Nunes, E.D. (2011) "Definição de Processos de Aquisição de Software para Reutilização", Dissertação de M.Sc., Programa de Pós-graduação em Engenharia de Sistemas e Computação - PESC/COPPE, Universidade Federal do Rio de Janeiro UFRJ Rio de Janeiro.

PMI (2008) "The Standard for Portfolio Management: Second Edition", Newtown Square, Project Management Institute - PMI.

Rocha, A.R., Santos, G., Barcellos, M.P. (2012) "Medição de Software e Controle Estatístico de Processos", Publicação do Ministério da Ciência, Tecnologia e Inovação, 2012.

SEI (2010) "CMMI® for Acquisition", Software Engineering Institute, Version 1.3, CMU/SEI-2010-TR-032.

Softex (2011) "Guia de Implementação - Parte 8: Implementação do MR-MPS:2011 (Níveis G a A) em organizações que adquirem software". 
TCU (2010) "Nota Técnica SEFTI/TCU nº 05, de 30 de abril de 2010. Condições em que há possibilidade de exigência da demonstração de qualidade de processo em contratações de serviços de software, a exemplo de CMMI e MPS.BR.", Tribunal de Contas da União, Secretaria de Fiscalização de Tecnologia da Informação - Sefti, Brasília.

Vargas, R.V. (2010) "Utilizando a Programação Multicritério (Analytic Hierarchy Process - AHP) para Selecionar e Priorizar Projetos na Gestão de Portfólio", PMI Global Congress 2010 - North America - Washington - DC - EUA - 2010. 